Draft Version OCtober 25, 2018

Preprint typeset using LATEX style emulateapj

\title{
MAGNETIC RECONNECTION TRIGGERED BY THE PARKER INSTABILITY IN THE GALAXY: TWO-DIMENSIONAL NUMERICAL MAGNETOHYDRODYNAMIC SIMULATIONS
AND APPLICATION TO THE ORIGIN OF X-RAY GAS IN THE GALACTIC HALO
}

\author{
Syuniti Tanuma ${ }^{1,2,3,4,5}$, TAkaAki Yokoyama, ${ }^{4,6}$, TAKahiro Kudoh ${ }^{4,7}$, \\ AND \\ KAZUNARI SHIBATA $1,4,8$ \\ Draft version October 25, 2018
}

\begin{abstract}
We propose the Galactic flare model for the origin of the X-ray gas in the Galactic halo. For this purpose, we examine the magnetic reconnection triggered by Parker instability (magnetic buoyancy instability), by performing the two-dimensional resistive numerical magnetohydrodynamic simulations. As a result of numerical simulations, the system evolves as following phases: Parker instability occurs in the Galactic disk. In the nonlinear phase of Parker instability, the magnetic loop inflates from the Galactic disk into the Galactic halo, and collides with the anti-parallel magnetic field, so that the current sheets are created in the Galactic halo. The tearing instability occurs, and creates the plasmoids (magnetic islands). Just after the plasmoid ejection, further current-sheet thinning occurs in the sheet, and the anomalous resistivity sets in. Petschek reconnection starts, and heats the gas quickly in the Galactic halo. It also creates the slow and fast shock regions in the Galactic halo. The magnetic field $(B \sim 3 \mu \mathrm{G})$, for example, can heat the gas $\left(n \sim 10^{-3} \mathrm{~cm}^{-3}\right)$ to temperature of $\sim 10^{6} \mathrm{~K}$ via the reconnection in the Galactic halo. The gas is accelerated to Alfvén velocity $\left(\sim 300 \mathrm{~km} \mathrm{~s}^{-1}\right)$. Such high velocity jets are the evidence of the Galactic flare model we present in this paper, if the Doppler shift of the bipolar jet is detected in the Galactic halo.
\end{abstract}

\section{Subject headings: Galaxy: halo — ISM: magnetic fields — instabilities —} magnetohydrodynamics

\section{INTRODUCTION}

The X-rays from hot gas are observed in the Galactic halo. Its luminosity and temperature are $L_{\mathrm{X}} \sim$ $7 \times 10^{39} \mathrm{erg} \mathrm{s}^{-1}$ and $T \sim 10^{6} \mathrm{~K}$ (Pietz et al. 1998). The volume and thermal energy are estimated to be $E \sim 10^{55} \mathrm{erg}$ and $V \sim 10^{68} \mathrm{~cm}^{3}$. To explain such hot gas in the Galactic halo, the "Galactic fountains" model has been proposed (i.e., supernova remnants and stellar winds heat the gas; Bregman 1980; Norman \& Ikeuchi 1989; Shapiro \& Benjamin 1991; Shull 1996; de Avillez 2000; Slavin, McKee, \& Hollenbach 2000). The energy source, however, may not be explained fully by this model because the evidence is not observed adequately (see also Birk, Lesch, \& Neukirch 1998). In this paper, we propose another mechanism. It is the Galactic flare model, i.e., the magnetic heating in the Galactic halo.

Parker (1992) pointed out the importance of magnetic reconnection for the heating of Galactic plasmas. Whenever the magnetic flux collides with another flux, which is not exactly parallel, the current density increases, and a strong dissipation sets in (e.g., via anomalous resistivity) to trigger fast reconnection (e.g., Ugai 1986). The magnetic reconnection is observed in solar flares by the X-ray satellites

\footnotetext{
${ }^{1}$ Kwazan Observatory, Kyoto University, Yamashina, Kyoto, 607-8471, Japan

2 tanuma@kwasan.kyoto-u.ac.jp

${ }^{3}$ Solar-Terrestrial Environment Laboratory, Nagoya University, 3-13 Honohara, Toyokawa, Aichi 442-8507, Japan

${ }^{4}$ National Astronomical Observatory of Japan, 2-21-1 Osawa, Mitaka, Tokyo 181-8588, Japan

${ }^{5}$ Department of Astronomy, School of Science, Tokyo University 7-3-1 Hongo, Bunkyo, Tokyo 113-0033, Japan

${ }^{6}$ Nobeyama Radio Observatory, Minamimaki, Minamisaku, Nagano 384-1305, Japan : yokoyama.t@nao.ac.jp

${ }^{7}$ Department of Physics and Astronomy, University of Western Ontario, London, Ontario N6A 3K7, Canada : kudoh@astro.uwo.ca

${ }^{8}$ shibata@kwasan.kyoto-u.ac.jp 
Yohkoh (Masuda et al. 1994; Shibata 1996; Tsuneta 1996) and SoHO (Yokoyama et al. 2001). In the solar flare, the reconnection heats the plasma from a temperature of $\sim$ several $\times 10^{6} \mathrm{~K}$ to $\sim$ several $\times 10^{7-8}$ $\mathrm{K}$, and accelerates it to Alfvén velocity $\left(\sim 10^{2-3}\right.$ $\mathrm{km} \mathrm{s}^{-1}$ ) (e.g., Shibata 1996). The reconnection would occur also in the Galaxy (Tanuma et al. 1999a, 1999b, 2001a, 1999b), which may be called "Galactic flare" (Sturrock \& Stern 1980; Kahn \& Brett 1993). Total magnetic energy is $E_{\mathrm{mag}} \sim\left(\langle B\rangle_{\mathrm{obs}}^{2} / 8 \pi\right) V_{\mathrm{G}}$ $\sim 10^{54.4} \mathrm{erg}$ at least, where $\langle B\rangle_{\text {obs }}(\sim 3 \mu \mathrm{G})$ is the mean observed field strength (see Beck et al. 1996; Vallée 1997), and $V_{\mathrm{G}}\left(\sim 10^{67} \mathrm{~cm}^{3}\right)$ is the volume of the Galaxy. The rotational energy of the Galaxy $\left(\sim 10^{58.9} \mathrm{erg}\right)$ and kinetic energy of the interstellar gas $\left(\sim 10^{58.2} \mathrm{erg}\right)$ are its origin (e.g., Parker 1971; Sturrock \& Stern 1980; Tanuma et al. 1999a; Tanuma 2000).

The steady reconnection mechanisms were proposed (see Priest \& Forbes 2000). In Sweet(1958)Parker(1957) type reconnection, the diffusion region is so long as to occupy whole current system. It can not be applied to the solar flare phenomena, because the reconnection rate of this model is too small $\left(\sim R_{\mathrm{m}}^{-1 / 2}\right)$ in the solar corona, where $R_{\mathrm{m}}$ is the magnetic Reynolds number $\left(\sim 10^{12}\right)$. On the other hand, in Petschek(1964) type reconnection, the diffusion region is localized near an X-point, and standing slow shocks occupy whole current systems. In this case, the energy conversion via slow shocks is much larger than Ohmic heating. This can hence be applicable to the solar flare phenomena, because the reconnection rate of this model is $\sim 0.1-0.01$. This is called "fast reconnection". A basic problem of Petschek model is as follows: Petschek reconnection occurs, if the anomalous resistivity sets in the current sheet (e.g., Ugai 1986; Yokoyama \& Shibata 1994; Tanuma 2000; Tanuma et al. 1999a, 2001a). The anomalous resistivity set in, when the current-sheet thickness becomes comparable with ion Lamor radius or ion inertial radius. It is, however, not fully known how the current sheet becomes thin, because the typical size of solar flare $\left(10^{9-11} \mathrm{~cm}\right)$ is much larger than these radii $\left(10^{2-3} \mathrm{~cm}\right)$. This situation is similar to that of the Galaxy $\left(R_{\mathrm{m}}>10^{15}\right)$, where typical size of magnetic field $\left(>10^{19} \mathrm{~cm}\right)$ is much larger than the ion Lamor radius $\left(\sim 10^{7} \mathrm{~cm}\right)$. To solve these problems, we proposed the current-sheet thinning via the "fractal tearing instability" (Tanuma 2000; Shibata \& Tanuma 2001; Tanuma et al. 2001a).

Many two-dimensional (2D) magnetohydrodynamic (MHD) numerical simulations have been carried out for the magnetic reconnection in the solar atmosphere (Magara \& Shibata 1997; Odstrčil \& Karlický 1997), and in the Galactic halo (Zimmer, Lesch,
\& Birk 1997; Birk et al. 1998), by assuming the current sheet at the initial condition (see also Nitta et al. 2001). Recently, Tanuma et al. (1999a, 1999b, 2001a) examined the magnetic reconnection triggered by a supernova-shock by performing the 2D MHD simulations with a high spatial resolution, and proposed that it can generate X-ray gas in the Galactic disk (e.g., Ebisawa et al. 2001). They found that the tearing instability (Furth, Killeen, \& Rosenbluth 1963) occurs in the current sheet long after the passage of a shock wave. Petschek reconnection occurs after further current sheet thinning via secondary tearing instability. In the present model, Parker(1966) instability creates the current sheet by itself and trigger the magnetic reconnection (Tanuma et al. 1999b; Tanuma 2000; see also Shibata, Nozawa, \& Matsumoto 1992; Yokoyama \& Shibata 1996, 1997).

Recently, we examied three-dimensional (3D) MHD simulations of the magnetic reconnection with a low spatial resolution. Petschek reconnection occurs after the current sheet thinning by the tearing instability in both 2D (Tanuma et al. 1999b) and 3D models (Tanuma 2000; Tanuma et al. 2001b), because we can not resolve the secondary tearing instability when we assume a rough grid (The similarities between 2D and 3D models are consistent with Ugai \& Shimizu 1996). 3D effect such as Rayleigh-Taylor instability, however, appears when reconnection jet collides with high pressure gas and magnetic loop much after the onset of Petschek reconnection. Tanuma et al. (2002) applied the results to the creation of helical magnetic field and confinement of high energy particles in the solar flare. We study the basic physics of magnetic reconnection which are common between $2 \mathrm{D}$ and $3 \mathrm{D}$ models, although 2D model examined in this paper is a toy model of limited in $2 \mathrm{D}$ dimension. In this paper, however, we examine the $2 \mathrm{D}$ model under a higher spatial resolution than the 3D model which we are able to do.

Parker instability is the undular mode $(\boldsymbol{k} \| \boldsymbol{B})$ of magnetic buoyancy instability (Parker 1966), which occurs if a gas layer in a gravitational field is supported by the horizontal magnetic fields. Suppose that the magnetic field lines are disturbed and begin to undulate. The gas in the loop top slides down along the field lines, so that loop rises further, and the instability sets in. Parker instability is suggested to influence the motion of clouds, HII regions, and OB associations (Tosa \& Sofue 1974), and the distribution of clouds (Mouschovias, Shu, \& Woodward 1974; Vrba 1977; Blitz \& Shu 1980; Elmegreen 1982); for example, Perseus hump (Sofue \& Tosa 1974), Perseus arm (Appenzeller 1971, 1974), Barnard loop (Mouschovias, Shu, \& Woodward 1974), and SofueHanda(1984) lobe. 
The linear analysis of Parker instability were made by many researchers (Shu 1974; Horiuchi et al. 1988; Hanawa et al. 1992; Foglizzo \& Taggar 1994; Chou et al. 1997; Kamaya et al. 1997). The 2D MHD simulations were performed for solar flares (Kaisig et al. 1990; Nozawa et al. 1992; Shibata et al. 1992; Yokoyama \& Shibata 1996), and the interstellar medium (Basu et al. 1997; Matsumoto et al. 1998; Santillán et al. 2000; Steinacker \& Shchekinov 2001). The three-dimensional (3D) simulations of Parker instability of horizontal magnetic field in solar atmosphere and Galaxy (Matsumoto et al. 1993; Kim, Ryu, \& Jones 2001; Hanasz, Otmianowska-Mazur, \& Lesch 2002), and the twisted flux tube in the solar atmosphere (Matsumoto et al. 1998; Abbett, Fisher, \& Fan 2000; Fan 2001; Magara 2001), the Galaxy (Hanasz \& Lesch 2000; France et al. 2002), and accretion disks (Ziegler 2001) are also performed. Shibata et al. (1989, 1992) and Yokoyama \& Shibata (1994, 1996, 1997) examined the magnetic reconnection triggered by Parker instability in the solar corona. Recently, Hanasz et al. (2002) examined the 3D model of the magnetic reconnection in magnetic loop created by Parker instability with Coriolis force in the interstellar medium. In the present paper, we extend Shibata et al. $(1989,1992)$ and Yokoyama \& Shibata (1994, 1996, 1997)'s solar flare model to the Galaxy: The Galactic flare as the origin of the X-ray gas in the Galactic halo.

In this paper, we propose a possible origin of $\mathrm{X}$ ray gas in the Galactic halo. In the next section, we describe the simulation method. In sections 3 and 4, we describe the results of numerical simulations, and discuss it. In the last section, we summarize this paper.

\section{NUMERICAL SIMULATIONS}

\subsection{The Situation of the Problem}

Figure 1 shows our schematic scenario. Figure $1 a$ displays the initial condition. Parker instability occurs in the Galactic disk (Fig. 1b; we call this situation phase I and II in this paper). The magnetic reconnection occurs and heats the gas (Fig. 1c; phase III) . The heated gas is confined by the magnetic field (Fig. 1d).

\subsection{Two-Dimensional Resistive MHD Basic Equations}

The resistive MHD basic equations are written as follows:

$$
\begin{aligned}
\frac{\partial \rho}{\partial t}+\nabla \cdot(\rho \boldsymbol{v}) & =0 \\
\rho \frac{\partial \boldsymbol{v}}{\partial t}+\rho(\boldsymbol{v} \cdot \nabla) \boldsymbol{v}+\nabla p_{g} & =\frac{1}{c} \boldsymbol{J} \times \boldsymbol{B}+\rho \boldsymbol{g},
\end{aligned}
$$

$$
\begin{aligned}
\frac{\partial \boldsymbol{B}}{\partial t}-\nabla \times(\boldsymbol{v} \times \boldsymbol{B}) & =-c \nabla \times(\eta \boldsymbol{J}), \\
\frac{\partial e}{\partial t}+\nabla \cdot\left[\left(e+p_{g}\right) \boldsymbol{v}\right] & =\eta|\boldsymbol{J}|^{2}+\boldsymbol{v} \cdot \nabla p_{g}
\end{aligned}
$$

where $\rho, \boldsymbol{v}, \boldsymbol{B}, \eta, e, \boldsymbol{J}$, and $\boldsymbol{g}$ are mass density, velocity, magnetic field, electric resistivity, internal energy, current density $(=c \nabla \times \boldsymbol{B} / 4 \pi)$, and gravity, respectively. We use the equation of state for the ideal gas, i.e., $p_{g}=(\gamma-1) e$, where $\gamma$ is the specific heat ratio $(=5 / 3)$. We take Cartesian coordinates $(x, z)$, where $x$ and $z$ axes are in horizontal and vertical directions, respectively. The velocity, magnetic field, and gravitational acceleration are $\boldsymbol{v}=\left(v_{x}, v_{z}\right), \boldsymbol{B}=\left(B_{x}, B_{z}\right)$, and $\boldsymbol{g}=[0,-g \tanh (z / 0.001)]$, where $g$ is constant.

\subsection{The Normalization}

We normalize the velocity, length, and time by the sound velocity $\left(C_{s}\right)$, gravitational scale height $(H)$, and $H / C_{s}$, respectively. The units of normalizations are

$$
\begin{aligned}
C_{s} & \sim 10 T_{4}^{1 / 2} \mathrm{~km} \mathrm{~s}^{-1} \\
H & \sim \frac{C_{s}^{2}}{g} \sim 100 T_{4}^{1 / 2} \mathrm{pc} \\
\tau & \equiv \frac{H}{C_{s}} \sim 10^{7} \mathrm{yr}
\end{aligned}
$$

where $T_{4}$ is the temperature in unit of $10^{4} \mathrm{~K}$, and $g$ is the acceleration of the gravity (Tanuma et al. 1999b; Tanuma 2000). The units of density, gas pressure, magnetic field strength, current density, and resistivity are $\rho_{0} \sim 1 \times 10^{-25} \mathrm{~g} \mathrm{~cm}^{-3}, \rho_{0} C_{s}^{2} \sim 4 \times 10^{-13}$ $\operatorname{erg~cm}{ }^{-3},\left(\rho_{0} C_{s}^{2} / 8 \pi\right)^{1 / 2} \sim 3.2 \mu \mathrm{G},\left(\rho_{0} C_{s}^{2} / 8 \pi\right)^{1 / 2} / H$, and $c^{2} H C_{s} / 4 \pi$, respectively. In this paper, we solve the non-dimensional basic equations, and describe the results of numerical simulations by non-dimensional values.

\subsection{The Anomalous Resistivity Model}

We assume the anomalous resistivity model as follows:

$$
\eta= \begin{cases}0 & \text { if } v_{d} \leq v_{c} \\ \alpha\left(v_{d} / v_{c}-1\right)^{2} & \text { if } v_{d}>v_{c}\end{cases}
$$

(e.g., Ugai 1986, 1992; Shibata et al. 1992; Yokoyama \& Shibata 1997; Ugai \& Kondoh 2001; Tanuma et al. 1999b; Tanuma 2000), where $v_{d}(\equiv|J| / \rho)$ and $v_{c}$ are the relative ion-electron drift velocity and threshold above which anomalous resistivity sets in. The parameters are $\alpha=0.01$ and $v_{c}=400$. 


\subsection{The Initial Condition of Typical Model}

For the initial condition, we assume that the cool, dense Galactic disk is between the hot, rarefied Galactic halo (Fig. 1a; Horiuchi et al. 1988; Matsumoto et al. 1988; Tanuma et al. 1999b; Tanuma 2000). Tables 1 and 2 show the variables and parameters. The temperature is

$$
T(x, z)=T_{0}+0.5\left[\tanh \left(\frac{z-z_{c}}{0.5}\right)+1.0\right]\left(T_{c}-T_{0}\right) .
$$

The parameters are $T_{0}=1, T_{c}=25$, and $z_{c}=6$. The basic physics does not much depend on $T_{c} / T_{0}$ (Matsumoto et al. 1988; Yokoyama \& Shibata 1996). The magnetic fields are in the Galactic disk $(|z|<$ $\left.z_{d}=2.5\right)$ and in the Galactic halo $\left(|z|>z_{h}=5\right)$. The initial magnetic field is horizontal $\left(B_{z}(x, z)=0\right)$ everywhere, so that the ratio of gas to magnetic pressure is $\beta_{0}=8 \pi p_{g}(x, z) / B_{x}(x, z)^{2}=0.2$. The $\beta(z)$ is

$$
\begin{aligned}
\frac{1}{\beta(z)} & =\frac{1}{2 \beta_{0}}\left[1-\tanh \left(\frac{z-z_{d}}{0.5}\right)\right] \\
& +\frac{1}{2 \beta_{0}}\left[1+\tanh \left(\frac{z-z_{h}}{0.5}\right)\right] .
\end{aligned}
$$

The value $\left(\beta_{0}=0.2\right)$ is attained in the Galactic disk, if the magnetic field of $B \sim 5^{1 / 2}\langle B\rangle_{\text {obs }} \sim 7 \mu \mathrm{G}$, where $\langle B\rangle_{\text {obs }}$ is the mean observed field strength $(\sim 3 \mu \mathrm{G})$ in the Galactic disk (see Beck et al. 1996; Vallée 1997). The magnetized gas is under the MHD equilibrium;

$$
\frac{d}{d z}\left[p_{g}(z)+\frac{B_{x}(z)^{2}}{8 \pi}\right]+\rho(z) g=0 .
$$

The variables are $\rho_{0}=1, p_{g 0}=0.6$, and $B_{x 0} \simeq 8.68$ at the equatorial plane. The sound and Alfvén velocity are $C_{s} \equiv\left(\gamma p_{g 0} / \rho_{0}\right)^{1 / 2}=1.0$ and $v_{\mathrm{A}}^{\text {init }}=$ $B_{x 0} /\left(4 \pi \rho_{0}\right)^{1 / 2} \simeq 2.45$ in the Galactic disk. The Galactic disk is unstable to Parker instability.

We use the $N_{x}=403$ grids and $N_{z}=604$ grids in the horizontal and vertical directions, respectively. The intervals of grids are uniform $(\triangle x=0.30)$ in $x$-axis and nonuniform $(\triangle z \geq 0.075)$ in $z$-axis. We assume the top $(z=+34.5)$ and bottom $(z=-34.5)$ surfaces are free boundaries, and the right $(x=$ $+30.0)$ and left $(x=-30.0)$ ones are periodic ones. We use the 2-steps modified Lax-Wendroff method. We put random perturbations $\left(<0.05 C_{s}\right)$ on vertical velocities $\left[v_{z}(x, z)\right]$ in the Galactic disk.

We neglect radiation and heat conduction. The cooling times due to the conduction and radiation for the cool $\left(T \sim 10^{4} \mathrm{~K}\right)$, dense $\left(n \sim 0.1 \mathrm{~cm}^{-3}\right)$ gas in the Galactic disk are

$$
\begin{aligned}
\tau_{\text {cond }} \sim & \frac{n k T \lambda^{2}}{\kappa_{0} T^{7 / 2}} \\
\sim & 10^{13}\left(\frac{n}{0.1 \mathrm{~cm}^{-3}}\right) \\
& \left(\frac{\lambda}{100 \mathrm{pc}}\right)^{2}\left(\frac{T}{10^{4} \mathrm{~K}}\right)^{-5 / 2} \mathrm{yr}, \\
\tau_{\text {rad }} \sim & \frac{n k T}{n^{2} \Lambda(T)} \\
\sim & 10^{3}\left(\frac{T}{10^{4} \mathrm{~K}}\right)\left(\frac{n}{0.1 \mathrm{~cm}^{-3}}\right)^{-1} \\
& {\left[\frac{\Lambda\left(10^{4} \mathrm{~K}\right)}{10^{-21} \mathrm{erg} \mathrm{cm}^{3} \mathrm{~s}^{-1}}\right]^{-1} \mathrm{yr} }
\end{aligned}
$$

respectively, where $\Lambda(T)$ is the cooling function (Spitzer 1978), and $\kappa_{0}$ is constant $\left(=10^{-6} \mathrm{erg} \mathrm{s}^{-1}\right.$ $\left.\mathrm{cm}^{-1} \mathrm{~K}^{-1}\right)$. For the hot $\left(T \sim 10^{6} \mathrm{~K}\right)$, rarefied $\left(n \sim 10^{-3} \mathrm{~cm}^{-3}\right)$ gas in the Galactic halo, however, they become

$$
\begin{aligned}
\tau_{\text {cond }} \sim & 10^{9}\left(\frac{n}{10^{-3} \mathrm{~cm}^{-3}}\right) \\
& \left(\frac{\lambda_{\text {eff }}}{3 \mathrm{kpc}}\right)^{2}\left(\frac{T}{10^{6} \mathrm{~K}}\right)^{-5 / 2} \mathrm{yr}, \\
\tau_{\text {rad }} \sim & 10^{9}\left(\frac{T}{10^{6} \mathrm{~K}}\right)\left(\frac{n}{10^{-3} \mathrm{~cm}^{-3}}\right)^{-1} \\
& {\left[\frac{\Lambda\left(10^{6} \mathrm{~K}\right)}{10^{-23} \mathrm{erg} \mathrm{cm}^{3} \mathrm{~s}^{-1}}\right]^{-1} \mathrm{yr}, }
\end{aligned}
$$

respectively, where $\lambda_{\text {eff }}$ is the effective length of helical magnetic loop. They are one order of magnitude longer than the typical time scale $\left(\sim 10^{8}\right.$ yr; see next section) of the physical process examined in this paper, so that the cooling mechanisms can be neglected, once they are heated to X-ray gas in the Galactic halo. The basic properties of the magnetic reconnection such as reconnection rate and energy release rate are not much affected by the heat conduction (Yokoyama \& Shibata 1997).

\section{THE RESULTS OF NUMERICAL SIMULATIONS}

\subsection{The typical Model (Model A1)}

\section{Phase I: The Linear Phase of Parker Insta-} bility $(t<40)$

Figures 2-4 show the time evolution of the system. The axes are in the unit of $\sim 10 H \sim 1$ kpc. The magnetic field lines starts to bent across the plane. Figures $2 a, 3 a$, and $4 a$ shows a characteristic feature of the odd-mode (glide-reflection mode), which grows earlier than even-mode (mirrorsymmetry mode) (Horiuchi et al. 1988; Matsumoto 
et al. 1988; see also Tanuma et al. 1999b; Tanuma 2000). The magnetic field inflates toward the Galactic halo by the magnetic buoyancy force. The gas slides down along the magnetic field lines.

Phase II: The Nonlinear Phase of Parker Instability $(t \sim 40-60)$

The system enters the nonlinear phase at $t \sim 40$. In the valleys of the waving field, the vertical dense spurs are formed almost perpendicular to the Galactic plane (Fig. 3a). The dense regions are also created in the valleys. Figure 5 shows the time variations of the drift velocity and velocity. The velocity increases to Alfvén velocity $(\sim 2.5-3.0)$ in this phase.

Figure 6 shows the time variation of the various energies. The magnetic, thermal, kinetic, gravitational, and total energies are defined by

$$
\begin{aligned}
E_{\mathrm{mag}} & =\iint \frac{B_{x}(x, z)^{2}+B_{z}(x, z)^{2}}{8 \pi} \mathrm{d} x \mathrm{~d} z \\
E_{\mathrm{th}} & =\iint \frac{1}{\gamma-1} p_{g}(x, z) \mathrm{d} x \mathrm{~d} z \\
E_{\text {kin }} & =\iint \frac{1}{2} \rho(x, z)\left(v_{x}(x, z)^{2}+v_{z}(x, z)^{2}\right) \mathrm{d} x \\
E_{\mathrm{g}} & =\iint \rho(x, z) g|z| \mathrm{d} x \mathrm{~d} z, \\
E_{\mathrm{tot}} & =E_{\mathrm{mag}}+E_{\mathrm{th}}+E_{\text {kin }}+E_{\mathrm{g}},
\end{aligned}
$$

respectively. They are integrated in whole simulation region, and corrected by the energy flux at the top and bottom boundaries. The gravitational and magnetic energies are converted to the thermal and kinetic ones.

The top of inflating loops collides with the antiparallel magnetic field in the Galactic halo (Figs. $2 b$ and $3 b$ ), so that the current sheets are created at $t \sim 60$ (Fig. $4 b$ ).

Phase III: The Magnetic Reconnection Triggered by Tearing Instability $(t>60)$

The drift velocity increases steeply at $t \sim 60$ (Fig. $5 a)$. The tearing instability occurs at the dissipation region, and creates the small magnetic islands (Tanuma 2000; Tanuma et al. 2001a). The islands are ejected along the current sheet. Just after the plasmoid ejection $(t \sim 62)$, the drift velocity increases above the threshold of anomalous resistivity. The anomalous resistivity sets in, and Petschek-like reconnection starts. The gas is accelerated horizontally to $\sim 8-13$ (Fig. $5 b$ ), which is equal to the local Alfvén velocity. The gas is heated to $T_{\max } \sim 125$ (Figs. $2 b$ and $2 c$ ). It is explained by

$$
T_{\max } \sim\left(1+\frac{1}{\beta}\right) \frac{n_{\text {in }}}{n_{\text {out }}} T_{\mathrm{in}},
$$

where $n_{\text {in }}, n_{\text {out }}$, and $T_{\text {in }}$ are the densities of inflowing and outflowing gas, and the temperature of inflowing gas. It is derived from the equation $n_{\text {out }} k T_{\max } \sim$ $n_{\text {in }} k T_{\text {in }}+B^{2} / 8 \pi$. We assume $\beta \sim \beta_{0} \sim 0.2, n_{\text {in }} \sim$ $n_{\text {out }}$, and $T_{\text {in }} \sim T_{c} \sim 25$.

Figures 7 and 8 show the slow and fast shock regions, respectively. Two peaks in the profile of current density are a characteristic pattern of the slow shock (see also Fig. 4c). The fast shock is also created by the collision between the reconnection jet and ambient high-pressure gas (Fig. 8).

The magnetic energy is released at the rate of $-d E_{\mathrm{mag}} / d t \sim 35$ (Fig. 6). It is determined by the Poynting flux entering into the reconnection region,

$$
-\frac{d E_{\mathrm{mag}}}{d t} \sim 2 \frac{B^{2}}{4 \pi} S v_{\text {in }} \sim \frac{B^{3} S}{4 \pi^{3 / 2} \rho^{1 / 2}}
$$

where $v_{\text {in }}\left(=\epsilon v_{\mathrm{A}}\right), \epsilon$, and $S$ are the inflow velocity, reconnection rate, and reconnection region size, respectively.

\subsection{The Parameter Survey}

\subsubsection{The Dependence on the Existence and Direction of the Magnetic Field in the Galactic Halo}

We examine the effect of the magnetic field in the Galactic halo, by comparing the anti-parallelfield model (typical model; model A1) with the nomagnetic-field model (model B), parallel-magneticfield model (model $\mathrm{C}$ ), and model D (anti-parallel magnetic field in a Galactic halo and parallel magnetic field in the other Galactic halo) (Table 1). In models $\mathrm{B}$ and $\mathrm{C}$, no magnetic reconnection occurs. In model $\mathrm{C}$, the parallel magnetic field in the Galactic halo suppresses Parker instability. The velocity is $\sim 3-6$, which is larger than that of model B (Fig. 5 ), because the gas is compressed between the magnetic loop and ambient magnetic field. In model D, the magnetic energy release rate is very small. On the other hand, the distribution of density do not depend on the magnetic reconnection.

\subsubsection{The Dependence on the Magnetic Field Strength}

We examine the dependence of results on $B_{x 0}[=$ $\left(8 \pi p_{g 0} / \beta_{0}\right)^{1 / 2}$ ], i.e., $\beta_{0}$ (models A2 and F1-9; Table $1)$. Figure $9 a$ shows the $\beta_{0}$-dependence of magnetic energy release rate. It is determined by the Poynting flux entering the reconnection region,

$$
\begin{aligned}
-\frac{d E_{\mathrm{mag}}}{d t} & \sim 2 \frac{B^{2}}{4 \pi} S v_{\mathrm{in}} \\
& \propto \beta_{0}^{-3 / 2},
\end{aligned}
$$


where $S$ is the reconnection region size, $v_{\text {in }}\left[=\epsilon v_{\mathrm{A}}=\right.$ $\left.\epsilon B /(4 \pi \rho)^{1 / 2}\right]$ is the inflow velocity to the reconnection region, and $\epsilon$ is the reconnection rate. It is also shown $\left(\sim 4.5 \beta_{0}^{-3 / 2}\right)$, by assuming $\epsilon \sim 0.1, S \sim 20$, and $p_{g} \sim 0.6$. It explains the results well. Figure $9 b$ shows the time when maximum heating rate is attained. It is determined by the time scale of Parker instability, i.e., Alfvén time $\left(\tau_{\mathrm{A}} \propto v_{\mathrm{A}}^{-1} \propto \beta_{0}^{1 / 2}\right)$, as shown $\left(\sim 100 v_{\mathrm{A}}^{-1} \sim 90 \beta_{0}^{1 / 2}\right)$, where we assume that the vertical of loop is $\sim 0.1 v_{\mathrm{A}}$ (Matsumoto et al. 1998) and the reconnection occurs at $z \sim 10$.

Figure $9 c$ shows the maximum temperature $\left(T_{\max }\right)$. The gas is heated to

$$
\begin{aligned}
T_{\max } & \sim\left(1+\frac{1}{\beta_{0}}\right) \frac{n_{\text {in }}}{n_{\text {out }}} T_{\text {in }} \\
& \propto 1+\frac{1}{\beta_{0}}
\end{aligned}
$$

(see eq. [23]), as also plotted $\left(\sim 25\left[1+\beta_{0}^{-1}\right]\right)$, by assuming $n_{\text {in }} \sim n_{\text {out }}$ and $T_{\text {in }} \sim T_{c} \sim 25$. It explains the results well. Figure $9 d$ shows the maximum velocity. The gas is accelerated to Alfvén velocity,

$$
\begin{aligned}
v_{\mathrm{jet}} \sim v_{\mathrm{A}} & =\frac{B}{(4 \pi \rho)^{1 / 2}} \\
& \propto \beta_{0}^{-1 / 2}
\end{aligned}
$$

as shown by assuming $T \sim 25$. It also explains the results well.

\subsubsection{The Dependence on the Magnetic Field in the Galactic Halo}

We examine the position $\left(z_{h}\right)$ of anti-parallel magnetic field (models A2 and G1-3; Table 1). In the near-magnetic-field model (model G3), the tearing instability occurs between the Galactic disk and halo. The magnetic field dissipates in an early phase $(t \sim$ $0-50)$, and the wavelength of the magnetic loops is shorter than that of the other models.

We also examine the existence of magnetic field in the Galactic halo, for the near-magnetic-field models (models G3, K, and L; Table 1). In models K (the nomagnetic-field model) and G3, the magnetic energy is released gradually by the tearing instability or magnetic dissipation in an early phase $(t \sim 0-35)$ (Figs. $10 a$ and $10 b$ ). In model L (the parallel-magnetic-field model), the dissipation is suppressed (Fig. 10c).

We, furthermore, examine $\beta_{0}$ in the near-magneticfield models (models G3 and J1-8; Table 1), as also shown in Figure 9 by $\triangle$. The $\beta_{0}$-dependence of these models are equal to that of high-magnetic-field models.

\subsubsection{The Dependence on the Resistivity Model}

We examine the resistivity model. Figure 11 shows the reconnection rate, in the anomalous (model A1) and the uniform resistivity model (models $\mathrm{M}$ and $\mathrm{N}$ ). In the uniform resistivity model, Sweet-Parker reconnection occurs, so that the reconnection rate is much smaller than that in the anomalous resistivity model. The reconnection rate increases transiently at $t \sim 40$. It is different from Yokoyama \& Shibata (1996)'s results: their reconnection rate is not timedependent. The reason is as follows; In our model, the position of magnetic field in the halo is lower than theirs, so that the magnetic field is stronger and the current density is larger at current sheet. The reconnection rate, however, stays at nearly steady small value, which is consistent with the Sweet-Parker scaling: $\eta|J| \propto \operatorname{Re}_{m}^{-1 / 2} \propto \eta^{1 / 2}$.

Table 3 shows the route to fast reconnection. In the anomalous resistivity model, Petschek type reconnection occurs after tearing instability and onsets of anomalous resistivity, while Sweet-Parker type reconnection occurs in the uniform resistivity model. Figure 12 displays the dependence on the resistivity models. It shows a characteristic patterns of two reconnection models.

\section{DISCUSSION}

\subsection{The Origin of X-Ray Gas in the Galactic Halo}

Parker instability is initiated by a small perturbation, a supernova explosion (Kamaya et al. 1997; Steinacker \& Shchekinov 2001), collision of the highvelocity clouds (HVCs; Santillán et al. 2000), and cosmic rays etc. The $\mathrm{X}$-ray gas can be generated, if the magnetic reconnection is triggered by Parker instability or the collision of HVCs (Kerp et al. 1994, 1996).

The reconnection heats the gas to

$$
T \sim 10^{6}\left(\frac{n}{10^{-3} \mathrm{~cm}^{-3}}\right)^{-1}\left(\frac{B}{3 \mu \mathrm{G}}\right)^{2} \mathrm{~K} .
$$

The reconnection also accelerates the gas to

$$
v_{\mathrm{A}} \sim 300\left(\frac{n}{10^{-3} \mathrm{~cm}^{-3}}\right)^{-1 / 2}\left(\frac{B}{3 \mu \mathrm{G}}\right) \mathrm{km} \mathrm{s}^{-1} .
$$

The duration of fast reconnection is

$$
\begin{aligned}
t \sim & \frac{l}{\epsilon v_{\mathrm{A}}} \\
\sim & 3000\left(\frac{l}{100 \mathrm{pc}}\right)\left(\frac{\epsilon}{0.1}\right)^{-1} \\
& \left(\frac{n}{10^{-3} \mathrm{~cm}^{-3}}\right)^{1 / 2}\left(\frac{B}{3 \mu \mathrm{G}}\right)^{-1} \mathrm{yr}
\end{aligned}
$$


where the reconnection rate is $\epsilon\left(=v_{\text {in }} / v_{\mathrm{A}}\right)$.

The anomalous resistivity sets in at least when the current sheet thickness becomes comparable with ion Lamor radius $\left[\sim 3 \times 10^{7}\left(T / 10^{4} \mathrm{~K}\right)^{1 / 2}(B / 3 \mu \mathrm{G})^{-1}\right.$ $\mathrm{cm}]$. The radius of magnetic island (i.e., plasmoid) is larger than it. The island in 2D simulation is helically twisted magnetic tube in $3 \mathrm{D}$. Its volume, mass, and luminosity are

$$
\begin{aligned}
& V_{\text {tube }} \sim 10^{61}\left(\frac{r_{p}}{10 \mathrm{pc}}\right)^{2}\left(\frac{l_{p}}{100 \mathrm{pc}}\right) \mathrm{cm}^{3}, \\
& M_{\text {tube }} \sim 2 \times 10^{33}\left(\frac{r_{p}}{10 \mathrm{pc}}\right)^{2}\left(\frac{l_{p}}{100 \mathrm{pc}}\right)\left(\frac{n}{1 \mathrm{~cm}^{-3}}\right) 36 \mathrm{~g} \\
& L_{\text {tube }} \sim n^{2} \Lambda(T) V_{\text {tube }} \\
& \sim 10^{34}\left(\frac{n}{10^{-3} \mathrm{~cm}^{-3}}\right)^{2}\left(\frac{\Lambda(T)}{10^{-21} \mathrm{erg} \mathrm{cm}^{3} \mathrm{~s}^{-1}}\right) \\
&\left(\frac{r_{p}}{10 \mathrm{pc}}\right)^{2}\left(\frac{l_{p}}{100 \mathrm{pc}}\right)
\end{aligned}
$$

where $r_{p}$ and $l_{p}$ are radius and length of the plasmoid, and $\Lambda(T)$ is the cooling function. Then, the number of magnetic tubes at such size is $\sim 10^{7-8}$.

Total released magnetic energy is

$$
E_{\mathrm{mag}} \sim 10^{51}\left(\frac{B}{3 \mu \mathrm{G}}\right)^{2}\left(\frac{\lambda_{\mathrm{tot}}}{1 \mathrm{kpc}}\right)^{2}\left(\frac{l}{100 \mathrm{pc}}\right) \mathrm{erg},
$$

where $\lambda_{\text {tot }}^{2}$ is the total area of many reconnection regions, and $l$ are the typical thickness of magnetic loop. The energy release rate, then, is

$$
\begin{aligned}
\frac{\mathrm{d} E_{\text {mag }}}{\mathrm{d} t} \sim & 10^{40}\left(\frac{B}{3 \mu \mathrm{G}}\right)^{3} \\
& \left(\frac{\lambda_{\text {tot }}}{1 \mathrm{kpc}}\right)^{2}\left(\frac{n}{10^{-3} \mathrm{~cm}^{-3}}\right)^{-1 / 2} \operatorname{erg~s}(39)
\end{aligned}
$$

It is also derived from eq. (26). It can explain the X-ray luminosity $\left(\sim 10^{39-40} \mathrm{erg} \mathrm{s}^{-1}\right.$; Pietz et al. 1998). The heated gas is confined for $\tau_{\text {cond }} \sim$ $10^{9}\left(n / 10^{-3} \mathrm{~cm}^{-3}\right)\left(\lambda_{\text {eff }} / 3 \mathrm{kpc}\right)^{2}\left(T / 10^{6} \mathrm{~K}\right)^{-5 / 2} \mathrm{yr}($ eq. [17]), where $\lambda_{\text {eff }}$ is the effective length of the helical magnetic tubes. The reconnection creates the bipolar jet, forming the high velocity gas at Alfvén velocity $\left(\sim 300 \mathrm{~km} \mathrm{~s}^{-1}\right.$; see also Nitta et al. 2001, Nitta, Tanuma, \& Maezawa 2002). The Doppler shift of the bipolar jet will be the evidence of the Galactic flare, proposed in this paper.

The plasmoid of cool gas is also created at the same time by the reconnection (Yokoyama \& Shibata 1996). It is also confined by magnetic field. The high velocity cool gas as well as hot gas will be the evidence of our model.

\subsection{The Comparison With Other Numerical Simulations}

The 2D model examined in this paper is an extension from the supernova-shock driven reconnection model in the Galactic disk (Tanuma et al. 1999a, 1999b, 2001a; Tanuma 2000). Some 2D numerical simulations were done for the reconnection in the Galaxy (Zimmer et al. 1997; Birk et al. 1998; Tanuma et al. 1999a, 2001a). They assume the current sheet for the initial condition (see also Ugai 1992; Ugai \& Kondoh 2001). Different from their models, Parker instability creates the current sheets spontaneously in our present model.

In our results, the tearing instability triggers Petschek reconnection. Sweet-Parker current sheet, however, would be created, and the secondary tearing instability will occur in the current sheet before Petschek reconnection, if we use fine grids like Tanuma et al. (1999a, 2001a)s' model (Table 3). Furthermore, in the actual Galaxy $\left(>10^{15}\right)$, as well as in solar corona $\left(\sim 10^{12}\right)$, the magnetic Reynolds number is much larger than that of the numerical simulations $\left(\sim 10^{4-5}\right)$, so that the current-sheet thickness must become much smaller than the currentsheet length, to set in the anomalous resistivity (e.g., through "fractal tearing instability"; Tanuma 2000; Shibata \& Tanuma 2001; Tanuma et al. 2001a).

A different situation from the solar flare model is the growth of odd-mode of Parker instability. Another different result from Yokoyama \& Shibata (1996)'s model is the time variation of reconnection rate in the uniform resistivity model (studied in section 3.2.4). On the other hand, in our model, Petschek reconnection occurs in the anomalous resistivity model, while Sweet-Parker reconnection occurs in the uniform resistivity model. This result is the same with Yokoyama \& Shibata (1994). We confirmed this in more general situation.

\subsection{Turbulence and $3 D$ Effects}

The MHD turbulence may be also important in reconnection problem (Lazarian \& Vishniac 1999). The effective reconnection rate increases if the diffusion region is in a state of MHD turbulence. It is, however, difficult to resolve MHD turbulence (even if in 2D).

Recently, we revealed that the fast reconnection occurs after the current sheet thinning by the tearing instability in both 2D (Tanuma et al. 1999b) and 3D models (Tanuma 2000; Tanuma et al. 2001b) under a low spatial resolution and the assumption of initial current sheet. We found no difference between 2D and $3 \mathrm{D}$ models in reconnection rate, inflow velocity, velocity of reconnection jet, temperature of heated gas, slow shock formation accompanied with Petschek 
reconnection, fast shock formation due to the collision between the reconnection jet and high pressure gas, and time scale of these phenomena. These results do not have a large quantitative difference from 2D models with a high spatial resolution examined by Tanuma et al. (2001a) (see also Ugai \& Shimizu 1996). Rayleigh-Taylor instability is, however, excited due to the collision, which occurs much after the onset of anomalous resistivity (Tanuma et al. 2002; in prep. for study of details).

We can not examine 3D model with the same small grid with that of $2 \mathrm{D}$ model, so that we examine $2 \mathrm{D}$ model with a small grid in this paper. The secondary tearing instability, however, may be different between $2 \mathrm{D}$ and $3 \mathrm{D}$, if we assume enough small grid. It is also important to study details of 3D structure of diffusion region, the tearing instability, plasmoid ejection, heating of X-ray gas in the Galactic halo, and creation of high velocity "bipolar jet", by performing $3 \mathrm{D}$ simulations with enough fine grid. It is our future work.

\section{SUMMARY}

We propose the Galactic flare model for the origin of the X-ray gas in the Galactic halo. We examine the magnetic reconnection triggered by Parker instability in the Galaxy, by performing the two-dimensional resistive MHD numerical simulations.

At the initial condition, we assume the horizontal flux sheet in the Galactic disk, and the anti-parallel magnetic field in the Galactic halo. The magnetic field inflates toward the Galactic halo, by Parker instability. It collides with the anti-parallel magnetic field in the Galactic halo, and creates the current sheets. The tearing instability occurs in the current sheet, and creates magnetic islands. Just after the plasmoid ejection, the anomalous resistivity sets in, and Petschek reconnection occurs. In the Galactic halo, the magnetic field of $B \sim$ several $\mu \mathrm{G}$ can heat the gas to $T \sim 10^{6} \mathrm{~K}$. If the bipolar jet of highvelocity hot gas or cool gas at Alfvén velocity $(\sim 300$ $\mathrm{km} \mathrm{s}^{-1}$ ) is observed, it is the evidence of the magnetic reconnection model in the Galactic halo.

The authors thank R. Matsumoto in Chiba University and K. Makishima in Tokyo University for fruitful discussions. The authors also gratefully acknowledge the constructive advice and useful comments of our referee toward improving our manuscript. The numerical computations were carried out on VPP5000 at the Astronomical Data Analysis Center of the National Astronomical Observatory, Japan, which is an inter-university research institute of astronomy operated by Ministry of Education, Culture, Sports, Science and Technology. This work was par- tially supported by Japan Science and Technology

Cooperation (ACT-JST). 


\section{REFERENCES}

Abbett, W. P., Fisher, G. H., \& Fan, Y. 2000, ApJ, 540, 548

Appenzeller, I. 1971 A\&A, 12, 313

Appenzeller, I. 1974 A\&A, 36, 99

Basu, S., Mouschovias, T. Ch., \& Paleologou, E. V. 1997, ApJ, 480, L'55

Beck, R., Brandenburg, A., Moss, D., Shukurov, A., \& Sokoloff, D. 1996, ARA\&A, 34, 155

Birk, G. T., Lesch, H., \& Neukirch, T. 1998, MNRAS, 296, 165

Blitz, L., \& Shu, F. H. 1980, ApJ, 238, 148

Bregman, J. N. 1980, ApJ, 236, 577

Chou, W., Tajima, T., Matsumoto, R., \& Shibata, K. 1997, PASJ, 49, 389

de Avillez, M. A. 2000, MNRAS, 315, 479

Ebisawa, K., Maeda, Y., Kaneda, H., \& Yamauchi, S. 2001, Science, 293, 1633

Elmegreen, B. G. 1982, ApJ, 253, 655

Fan Y. 2001, ApJ, 554, L111

Foglizzo, T., \& Tagger, M. 1994, A\&A, 287, 297

Franco, J., Kim, J., Alfaro, E. J., \& Hong, S. S. 2002, ApJ, 570, 647

Furth, H. P., Killeen, J, \& Rosenbluth, M. N. 1963, Phys. of Fluid, 6, 459

Hanawa, T., Matsumoto, R., \& Shibata, K. 1992, ApJ, 393, L71

Hanasz, M., \& Lesch, H. 1998, A\&A, 332, 77

Hanasz, M., \& Lesch, H. 2000, ApJ, 543, 235

Hanasz, M., Otmianowska-Mazur, K., \& Lesch H., 2002, A\&A386, 347

Horiuchi, T., Matsumoto, R., Hanawa, T., \& Shibata, K. 1988, PASJ, 40, 147

Kahn, F. D.. \& Brett, L. 1993, MNRAS, 263, 37

Kaisig, M., Tajima, T., Shibata, K., Nozawa, S., \& Matsumoto, R., 1990, ApJ, 358, 698

Kamaya, H, Horiuchi, T., Matsumoto, R., Hanawa, T., Shibata, K., \& Mineshige, S. 1997, ApJ, 486, 307

Kerp, J., Lesch, H., \& Mack, K.-M. 1994, A\&A, 286, L13

Kerp, J., Mack, K.-H., Egger, R., Pietz, J., Zimmer, F., Mebold, U., Burton, W. B., \& Hartmann, D. 1996, A\&A, 312, 67

Kim, E., Ryu, D., \& Jones, T. W. 2001, ApJ, , 557, 464

Lazarian, A. \& Vishniac, E. T. 1999, ApJ, 517, 700

Magara, T. \& Shibata, K. 1997, ApJ, 514, 456

Magara, T. 2001, ApJ, 549, 608

Masuda, S., Kosugi, T., Hara, H., Tsuneta, S., \& Ogawara, Y. 1994, Nature, 371, 495

Matsumoto, R., Horiuchi, T., Shibata, K., \& Hanawa, T. 1988, PASJ, 40, 171

Matsumoto, R., Tajima, T., Shibata, K., \& Kaisig, M. 1993, ApJ, 414, 357,

Matsumoto, R., Tajima, T., Chou, W., Okubo, A., Shibata, K. 1998, ApJ, 493, 43

Mouschovias, T. C., Shu, F. H., \& Woodward, P. R. 1974, A\&A, 33, 73

Nitta, S., Tanuma, S., Shibata, K., \& Maezawa, K. 2001, ApJ, 550,1119

Nitta, S., Tanuma, S., \& Maezawa, K. 2002, ApJ, in press (astro-ph/0208121)

Norman, C. A.. \& Ikeuchi, S. 1989, ApJ, 345, 372,

Nozawa, S., Shibata, K., Matsumoto, R., Sterling, A. C., Tajima, T., Uchida, Y., Ferrari, A., \& Rosner, R. 1992, ApJS, 78,267

Odstrčil, D., \& Karlický, M. 1997, A\&A, 326, 1252

Parker, E. N. 1957, J. Geophys. Res., 62, 509

Parker, E. N. 1966, ApJ, 145, 811

Parker, E. N. 1971, ApJ, 163, 255
Parker, E. N. 1990, IAU Symp., 140, 169

Parker, E. N. 1992, ApJ, 401, 137

Petschek, H. E. 1964, in AAS-NASA Symp. on the Physics of Solar Flares, ed. W. N. Hess (Washington DC: NASA), 425

Pietz, J., Kerp, J., Kalberla, P. M. W., Burton, W. B., Hartmann, D. \& Mebold, U. 1998, A\&A, 332, 55

Priest, E. \& Forbes, T. 2000, Magnetic reconnection : MHD theory and applications, (New York: Cambridge University Press)

Raymond, J. C. 1992, ApJ, 384, 502

Santillán, A., Kim, J., Franco, J., Martos, M., Hong, S. S., \& Ryu, D. 2000, ApJ, 545, 353

Shapiro P. R., \& Benjamin, R. A. 1991, PASP, 103, 923

Shibata, K., Tajima, T., Steinolfson, R. S., \& Matsumoto, R., 1989, ApJ, 345, 584

Shibata, K. \& Matsumoto, R. 1991, Nature, 353, 633

Shibata, K., Nozawa, S., \& Matsumoto, R. 1992, PASJ, 44, 265

Shibata, K. 1996, Adv. Space Res., 17, 9

Shibata, K., \& Tanuma, S. 2001, Earth, Planet and Space, 53, 473

Shu, F. 1974, A\&A, 33, 55

Shull, J. M. 1996, Nature, 380, 668

Slavin, J. D., McKee, C. F., \& Hollenbach, D. J. 2000 ApJ, 541,218

Sofue, Y., \& Handa, T. 1984, Nature, 310, 568

Sofue, Y., Klein, U., Beck, R., \& Wielebinski, R. 1986, ARA\&A, 24. 456

Sofue, Y., \& Tosa, M. 1974, A\&A, 36, 237

Spitzer, L. 1978, Physical Processes in the Interstellar Medium (New York: John Wiley \& Sons), ch 6

Steinacker, A. \& Shchekinov, Y. A. 2001, MNRAS, 325, 208

Sturrock, P. A., \& Stern, R. 1980, ApJ, 238, 98

Sweet, P. A. 1958, in Electromagnetic Phenomena in Cosmical Physics, IAU Symp. 6, ed. B. Lehnert (Boston: Kluwer), 123

Tanuma, S., Yokoyama, T., Kudoh, T., Matsumoto, R., Shibata, K., \& Makishima, K. 1999a, PASJ, 51, 161

Tanuma, S., Y̌okoyama, T., Kudoh, T., \& Shibata, K. 1999b, in Numerical Astrophysics, ed. S. M. Miyama, K. Tomisaka, \& T. Hanawa (New York: Kluwer), 119

Tanuma, S. 2000, Ph.D. thesis, Univ. Tokyo

Tanuma, S., Yokoyama, T., Kudoh, T., \& Shibata, K. 2001a, ApJ, 551, 312

Tanuma, S., Yokoyama, T., Kudoh, T., \& Shibata, K. 2001b, J. Korean Astron. Soc., 34, 309

Tanuma, S., Yokoyama, T., Kudoh, T., \& Shibata, K. 2002, COSPAR Corolquia Ser. 13, ed.' P. C. H. Martens \& D. Cauffman, in press

Tosa, M., \& Sofue, Y. 1974, A\&A, 1974, 32, 461

Tsuneta, S. 1996, ApJ, 456, L63

Ugai, M. 1986, Phys. of Fluid, 29, 3659

Ugai, M. 1992, Phys. of Fluid, 4, 2953

Ugai, M. \& Shimizu, T. 1996, Phys. of Plasmas, 3, 853

Ugai, M. \& Kondoh, K. 2001, Phys. of Plasmas, 8, 1545

Vallée, J. P. 1997, Fund. Cosmic Phys., 19, 1

Vrba, F. J. 1977, ApJ, 82, 198

Yokoyama, T., \& Shibata, K. 1994, ApJ, 436, L97

Yokoyama, T., \& Shibata, K. 1996, PASJ, 48, 353

Yokoyama, T., \& Shibata, K. 1997, ApJ, 474, L61

Yokoyama, T., Akita, K., Morimoto, T., Inoue, K., \& Newmark, J. 2001, ApJ, 546, L69

Zimmer, F., Lesch, H., \& Birk, G. T. 1997, A\&A, 320, 746

Ziegler, U. 2001, A\&A, 367, 170 
TABLE 1

Parameters. The typical model is named A1. The Unit of Length is $\sim 100$ PC.

\begin{tabular}{|c|c|c|c|c|c|c|c|c|c|}
\hline model & $\beta_{0}^{\mathrm{a}}$ & $z_{h}^{\mathrm{b}}$ & $z_{c}{ }^{\mathrm{c}}$ & $B_{u}^{\mathrm{d}}$ & $B_{l}{ }^{\mathrm{e}}$ & $\triangle x^{\mathrm{f}} \times \triangle z^{\mathrm{g}}$ & $N_{x}^{\mathrm{h}} \times N_{z}^{\mathrm{i}}$ & $L_{x}^{\mathrm{j}} \times L_{z}^{\mathrm{k}}$ & $\eta^{1}$ \\
\hline $\mathrm{A} 1$ & 0.200 & 5.0 & 6.0 & $\uparrow \downarrow$ & $\uparrow \downarrow$ & $0.300 \times 0.075$ & $403 \times 603$ & $120 \times 69.2$ & anomalous \\
\hline A2 & 0.200 & 5.0 & 6.0 & $\uparrow \downarrow$ & $\uparrow \downarrow$ & $0.350 \times 0.200$ & $203 \times 303$ & $70 \times 60.0$ & anomalous \\
\hline B & 0.200 & - & 6.0 & none & none & $0.300 \times 0.075$ & $403 \times 603$ & $120 \times 69.2$ & anomalous \\
\hline $\mathrm{C}$ & 0.200 & 5.0 & 6.0 & $\uparrow \uparrow$ & $\uparrow \uparrow$ & $0.300 \times 0.075$ & $403 \times 603$ & $120 \times 69.2$ & anomalous \\
\hline $\mathrm{D}$ & 0.200 & 5.0 & 6.0 & $\uparrow \downarrow$ & $\uparrow \uparrow$ & $0.300 \times 0.075$ & $403 \times 603$ & $120 \times 69.2$ & anomalous \\
\hline F1 & 0.100 & 5.0 & 6.0 & $\uparrow \downarrow$ & $\uparrow \downarrow$ & $0.350 \times 0.200$ & $203 \times 303$ & $70 \times 60.0$ & anomalous \\
\hline F2 & 0.175 & 5.0 & 6.0 & $\uparrow \downarrow$ & $\uparrow \downarrow$ & $0.350 \times 0.200$ & $203 \times 303$ & $70 \times 60.0$ & anomalous \\
\hline F3 & 0.225 & 5.0 & 6.0 & $\uparrow \downarrow$ & $\uparrow \downarrow$ & $0.350 \times 0.200$ & $203 \times 303$ & $70 \times 60.0$ & anomalous \\
\hline F4 & 0.250 & 5.0 & 6.0 & $\uparrow \downarrow$ & $\uparrow \downarrow$ & $0.350 \times 0.200$ & $203 \times 303$ & $70 \times 60.0$ & anomalous \\
\hline F5 & 0.275 & 5.0 & 6.0 & $\uparrow \downarrow$ & $\uparrow \downarrow$ & $0.350 \times 0.200$ & $203 \times 303$ & $70 \times 60.0$ & anomalous \\
\hline F6 & 0.300 & 5.0 & 6.0 & $\uparrow \downarrow$ & $\uparrow \downarrow$ & $0.350 \times 0.200$ & $203 \times 303$ & $70 \times 60.0$ & anomalous \\
\hline F7 & 0.500 & 5.0 & 6.0 & $\uparrow \downarrow$ & $\uparrow \downarrow$ & $0.350 \times 0.200$ & $203 \times 303$ & $70 \times 60.0$ & anomalous \\
\hline F8 & 1.000 & 5.0 & 6.0 & $\uparrow \downarrow$ & $\uparrow \downarrow$ & $0.350 \times 0.200$ & $203 \times 303$ & $70 \times 60.0$ & anomalous \\
\hline F9 & 1.200 & 5.0 & 6.0 & $\uparrow \downarrow$ & $\uparrow \downarrow$ & $0.350 \times 0.200$ & $203 \times 303$ & $70 \times 60.0$ & anomalous \\
\hline G1 & 0.200 & 6.0 & 7.0 & $\uparrow \downarrow$ & $\uparrow \downarrow$ & $0.350 \times 0.200$ & $203 \times 303$ & $70 \times 60.0$ & anomalous \\
\hline G2 & 0.200 & 4.5 & 5.5 & $\uparrow \downarrow$ & $\uparrow \downarrow$ & $0.350 \times 0.200$ & $203 \times 303$ & $70 \times 60.0$ & anomalous \\
\hline G3 & 0.200 & 4.0 & 5.0 & $\uparrow \downarrow$ & $\uparrow \downarrow$ & $0.350 \times 0.200$ & $203 \times 303$ & $70 \times 60.0$ & anomalous \\
\hline J1 & 0.175 & 4.0 & 5.0 & $\uparrow \downarrow$ & $\uparrow \downarrow$ & $0.350 \times 0.200$ & $203 \times 303$ & $70 \times 60.0$ & anomalous \\
\hline $\mathrm{J} 2$ & 0.180 & 4.0 & 5.0 & $\uparrow \downarrow$ & $\uparrow \downarrow$ & $0.350 \times 0.200$ & $203 \times 303$ & $70 \times 60.0$ & anomalous \\
\hline $\mathrm{J} 3$ & 0.225 & 4.0 & 5.0 & $\uparrow \downarrow$ & $\uparrow \downarrow$ & $0.350 \times 0.200$ & $203 \times 303$ & $70 \times 60.0$ & anomalous \\
\hline $\mathrm{J} 4$ & 0.250 & 4.0 & 5.0 & $\uparrow \downarrow$ & $\uparrow \downarrow$ & $0.350 \times 0.200$ & $203 \times 303$ & $70 \times 60.0$ & anomalous \\
\hline J5 & 0.275 & 4.0 & 5.0 & $\uparrow \downarrow$ & $\uparrow \downarrow$ & $0.350 \times 0.200$ & $203 \times 303$ & $70 \times 60.0$ & anomalous \\
\hline J6 & 0.300 & 4.0 & 5.0 & $\uparrow \downarrow$ & $\uparrow \downarrow$ & $0.350 \times 0.200$ & $203 \times 303$ & $70 \times 60.0$ & anomalous \\
\hline J7 & 0.500 & 4.0 & 5.0 & $\uparrow \downarrow$ & $\uparrow \downarrow$ & $0.350 \times 0.200$ & $203 \times 303$ & $70 \times 60.0$ & anomalous \\
\hline J8 & 1.000 & 4.0 & 5.0 & $\uparrow \downarrow$ & $\uparrow \downarrow$ & $0.350 \times 0.200$ & $203 \times 303$ & $70 \times 60.0$ & anomalous \\
\hline $\mathrm{K}$ & 0.200 & - & 5.0 & none & none & $0.350 \times 0.200$ & $203 \times 303$ & $70 \times 60.0$ & anomalous \\
\hline $\mathrm{L}$ & 0.200 & 4.0 & 5.0 & $\uparrow \uparrow$ & $\uparrow \uparrow$ & $0.350 \times 0.200$ & $203 \times 303$ & $70 \times 60.0$ & anomalous \\
\hline $\mathrm{M}$ & 0.200 & 5.0 & 5.0 & $\uparrow \downarrow$ & $\uparrow \downarrow$ & $0.300 \times 0.075$ & $403 \times 603$ & $120 \times 69.2$ & $\operatorname{uniform}(\eta=0.05)$ \\
\hline $\mathrm{N}$ & 0.200 & 5.0 & 5.0 & $\uparrow \downarrow$ & $\uparrow \downarrow$ & $0.300 \times 0.075$ & $403 \times 603$ & $120 \times 69.2$ & $\operatorname{uniform}(\eta=0.10)$ \\
\hline
\end{tabular}

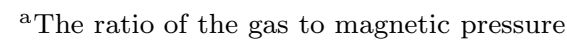

${ }^{\mathrm{b}}$ The position of magnetic field in the Galactic halo

${ }^{\mathrm{c}}$ The position of hot gas in the Galactic halo $\left(z_{c}=z_{h}+1\right)$

$\mathrm{d}$ The magnetic field in the upper corona ( $\uparrow:$ parallel, $\uparrow \downarrow$ :anti-parallel)

e The magnetic field in the lower corona ( $\uparrow:$ parallel, $\uparrow \downarrow$ :anti-parallel)

${ }^{\mathrm{f}}$ The grid size in $x$-axis (uniform)

${ }^{\mathrm{g}}$ Minimum grid size in $z$-axis

${ }^{\mathrm{h}}$ The number of grids in $x$-axis

${ }^{\text {i }}$ The number of grids in $z$-axis

jThe simulation region size in $x$-axis

${ }^{\mathrm{k}}$ The simulation region size in $z$-axis

${ }^{1}$ The resistivity model 
TABle 2

VARIABles AT THE INITIAL CONDITION FOR THE TYPICAL MODEL (MODEL A1).

\begin{tabular}{ccccc}
\hline \hline variable & cool dense disk & \multicolumn{3}{c}{ hot rarefied halo } \\
\hline$T$ & $T_{0}=1$ & $\left(|z|<z_{c}\right)$ & $T=25 T_{0}$ & $\left(|z|>z_{c}\right)$ \\
$\rho$ & $\rho_{0}=1$ & $(|z|=0)$ & $\rho \sim 0.004 \rho_{0}$ & $\left(|z| \sim z_{h}\right)$ \\
$p_{g}$ & $p_{g 0}=0.6$ & $(|z|=0)$ & $p_{g} \sim 0.1 p_{g 0}$ & $\left(|z| \sim z_{h}\right)$ \\
$B_{x}$ & $B_{x 0} \sim 8.68$ & $(|z|=0)$ & $B_{x} \sim 0.3 B_{x 0}$ & $\left(|z| \sim z_{h}\right)$ \\
$p_{\text {tot }}$ & $p_{\text {tot }, 0}=3.6$ & $(|z|=0)$ & $p_{\text {tot }} \sim 0.1 p_{\text {tot }, 0}$ & $\left(|z| \sim z_{h}\right)$ \\
$\beta_{0}$ & $\beta_{0}=0.2$ & $(|z|=0)$ & $\beta_{0}=0.2$ & $\left(|z|>z_{h}\right)$ \\
\hline
\end{tabular}


TABLE 3

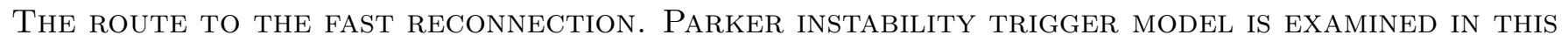

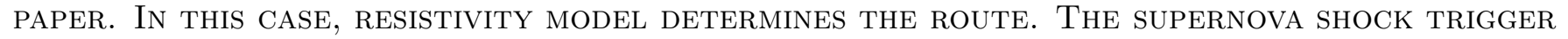
MODEL is EXAMINED in TANUMA ET AL. (2001A).

\begin{tabular}{llll}
\hline \hline & Parker instability trigger & Parker instability trigger & supernova shock trigger \\
\hline trigger & Parker instability & Parker instability & supernova shock \\
resistivity model & anomalous & uniform & anomalous \\
\hline evolution & downflow along rising loop & downflow along rising loop & tearing instability \\
& sheet thinning & sheet thinning & sheet thinning \\
& $\downarrow$ & Sweet-Parker sheet & Sweet-Parker sheet \\
& tearing instability & - & secondary tearing \\
& further sheet thinning & - & further sheet thinning \\
& anomalous resistivity & - & anomalous resistivity \\
& Petschek reconnection & - & Petschek reconnection \\
\hline
\end{tabular}


Fig. 1. - Schematic illustration of our numerical simulations. (a) As the initial condition, we assume the horizontal, parallel magnetic field and cool, dense gas in the Galactic disk, and the nearly uniform magnetic field which is anti-parallel to the disk field and hot, rarefied gas in the Galactic halo. The anti-parallel field is created, for example, by Coliori's force. (b) Parker instability is initiated by the random perturbations in the Galactic disk. The magnetic field in the Galactic disk bends across the equatorial plane. (c) The inflating magnetic loop collides with the anti-parallel magnetic field in the Galactic halo. The magnetic reconnection occurs. (d) The heated gas is confined by the magnetic field in the Galactic halo for a long time.

FIG. 2.- The temperature $(T)$ in the unit of $T_{0} \sim 10^{4} \mathrm{~K}$, magnetic field lines, and velocity vectors, for a typical model (model A1), The units of length, velocity, and time are $1 \mathrm{kpc}, 10 \mathrm{~km} \mathrm{~s}^{-1}, 10^{7} \mathrm{yr}$, respectively. (a) Parker instability occurs in the Galactic disk. The magnetic field of the Galactic disk inflates to the Galactic halo. (b) The inflating magnetic loop collides with anti-parallel magnetic field in the Galactic halo. The current sheets are created. (c) The magnetic reconnection occurs at $t \sim 62$, and heats the gas to $T_{\max } \sim 125$. (d) The heated gas is confined by the magnetic field in the Galactic halo.

Fig. 3. - Same with Figure 2, but the density $(\rho)$ in the unit of $10^{-25}$ g. (b) The gas, which is initially between the Galactic disk and halo, are raised by the inflating magnetic loops. (d) The large high-density clouds are created at the valleys of magnetic loops, and will become star forming regions.

FIG. 4.- Same with Figure 2, but the current density $(J)$. (b) Eight magnetic loops collide with anti-parallel magnetic field in the Galactic halo, so that the current density increases there. (d) The magnetic field confines the high-current-density regions at the valleys of magnetic loops near the Galactic plane.

FIG. 5.- Time variations of (a) drift velocity $\left(v_{d}=|J| / \rho\right)$ and (b) velocity $\left(\left|v_{x}\right|\right)$, for model A1 (solid lines; typical model, i.e., anti-parallel-magnetic-field model), model B (dotted lines; the no-magnetic-field model), model C (dashed lines; the parallelmagnetic-field model), and model D (dashed and dotted lines; the anti-parallel magnetic field in a halo and parallel magnetic field in the other halo). (a) In models A1 and D, the drift velocity increases steeply above the threshold $\left(v_{c}=400\right)$ of anomalous resistivity at $t \sim 62-65$. (b) In all models, the velocity increases gradually by Parker instability to $\sim 2.5-3(t \sim 40-62)$. In models A1 and D, by the magnetic reconnection $(t>62)$, the velocity increases steeply to $\sim 8-13$, which is equal to the local Alfvén velocity in the Galactic halo.

FIG. 6. - Time variations of the various energies. (a) The gravitational energy is released by Parker instability in its nonlinear phase $(t \sim 40-62)$. The magnetic energy is released mainly by the magnetic reconnection $(t>62)$. (b) The magnetic energy release rate increases to $-d E_{\mathrm{mag}} / d t \sim 75$ by the magnetic reconnection $(t \sim 70)$.

FIG. 7. - The profile of the variables in $x=-13.8$ at $t=70$. The magnetic reconnection occurs around $(x, z) \sim(-26,7.8)$. The fast reconnection such as Petschek model is accompanied by two slow shock regions along the reconnection jets. The profile of current density $(|J|)$ has two peaks at two slow shock regions.

FIG. 8. - Same with Figure7, but $z=6.5$. The gas is accelerated, by magnetic tension force, to the local Alfvén velocity in the Galactic halo, and collides with the ambient gas. The fast shock region is created around $x \sim-10$.

Fig. 9. - The dependence of the results on $\beta_{0}$, i.e., the magnetic field strength $\left[B_{x}=\left(8 \pi p_{g} / \beta_{0}\right)^{1 / 2}\right]($ models A2, F1-0, G3, and J1-8). The results of the far-magnetic-field models (models A2 and F1-9) and the near-magnetic-field models (models G3 and J1-8) are shown by $\diamond$ (with the dashed lines) and $\triangle$ (with the solid lines), respectively. (a) The maximum magnetic energy release rate $\left(-d E_{\mathrm{mag}} / d t\right)$. It is determined by Poynting flux $\left(\propto \beta_{0}^{-3 / 2}\right.$; the dashed line). (b) The time when the maximum magnetic energy release rate is attained. The time scales of these phenomena depend on Alfvén time $\left(\propto v_{\mathrm{A}}^{-1} \propto \beta_{0}^{1 / 2}\right.$; the dashed lines). (c) The maximum temperature $\left(T_{\max }\right)$ of heated gas. It is determined by the released magnetic energy $\left(\propto 1+1 / \beta_{0}\right.$; the dashed line). (d) Maximum $v_{x}$, which is determined by Alfvén velocity $\left(v_{\mathrm{A}} \propto \beta_{0}^{-1 / 2}\right.$; the dashed line).

FIG. 10. - Time variations of the various energies, for the near-magnetic-field models (models G3, K, and L). (a) In model G3 (the anti-parallel-magnetic-field model), the magnetic field dissipates by the tearing instability or magnetic dissipation $(t \sim 0-40)$. The wavelength of the magnetic loops is shorter than that of the typical model. (b) In model K (the no-magnetic-field model), the magnetic dissipation occurs in an early phase. (c) In model L (the parallel-magnetic-field model), the magnetic field does not dissipate in an early phase, because the parallel field of the Galactic halo suppresses the dissipation.

FIG. 11. - The time variation of the reconnection rate $(\eta|J|)$, in the anomalous resistivity model (model A1) and the uniform resistivity model $(\eta=0.05$ [the dashed line; model M] and $\eta=0.10$ [the dotted line; model N]). Petschek reconnection occurs in the anomalous resistivity model. On the other hand, Sweet-Parker reconnection occurs, in the uniform resistivity model.

FIG. 12. - The current density, magnetic field lines, and velocity vectors, for (a) the anomalous resistivity model (model A1) and (b) the uniform resistivity model $(\eta=0.05$; model M]). (a) Petschek reconnection occurs in the anomalous resistivity model. The small dissipation region and slow shock regions are the characteristics of Petschek reconnection. (b) In the uniform resistivity model, the long current sheet forms because Sweet-Parker reconnection occurs. 
This figure "f1.jpg" is available in "jpg" format from: http://arxiv.org/ps/astro-ph/0209008v1 
This figure "f2.jpg" is available in "jpg" format from: http://arxiv.org/ps/astro-ph/0209008v1 
This figure "f3.jpg" is available in "jpg" format from: http://arxiv.org/ps/astro-ph/0209008v1 
This figure "f4.jpg" is available in "jpg" format from: http://arxiv.org/ps/astro-ph/0209008v1 
This figure "f5.jpg" is available in "jpg" format from: http://arxiv.org/ps/astro-ph/0209008v1 
This figure "f6.jpg" is available in "jpg" format from: http://arxiv.org/ps/astro-ph/0209008v1 
This figure "f7.jpg" is available in "jpg" format from: http://arxiv.org/ps/astro-ph/0209008v1 
This figure "f8.jpg" is available in "jpg" format from: http://arxiv.org/ps/astro-ph/0209008v1 
This figure "f9.jpg" is available in "jpg" format from: http://arxiv.org/ps/astro-ph/0209008v1 
This figure "f10.jpg" is available in "jpg" format from: http://arxiv.org/ps/astro-ph/0209008v1 
This figure "f11.jpg" is available in "jpg" format from: http://arxiv.org/ps/astro-ph/0209008v1 
This figure "f12.jpg" is available in "jpg" format from: http://arxiv.org/ps/astro-ph/0209008v1 